Please do not remove this page

RMIT

UNIVERSITY

\title{
Structure of the Janus Protein Human CLIC2
}

Cromer, Brett; Gorman, Michael; Hansen, Guido; Adams, Julian; Coggan, Marjorie; Littler, Dene; Brown, Louise

https://researchrepository.rmit.edu.au/esploro/outputs/9921858170201341/filesAndLinks?institution=61RMIT_INST\&index=null

Cromer, B., Gorman, M., Hansen, G., Adams, J., Coggan, M., Littler, D., Brown, L., Mazzanti, M., Breit, S., Curmi, P., Dulhunty, A., Board, P., \& Parker, M. (2007). Structure of the Janus Protein Human CLIC2. Journal of Molecular Biology, 373(3), 719-731. https://doi.org/10.1016/j.jmb.2007.09.041 Document Version: Accepted Manuscript

Published Version: https://doi.org/10.1016/j.jmb.2007.09.041

Repository homepage: https://researchrepository.rmit.edu.au (c) 2007 Elsevier Ltd. All rights reserved

Downloaded On 2023/04/26 21:18:50 +1000 
Cromer, B, Gorman, M, Hansen, G, Adams, J, Coggan, M, Littler, D, Brown, L, Mazzanti, M, Breit, S, Curmi, P, Dulhunty, A, Board, P and Parker, M 2007, 'Structure of the Janus Protein Human CLIC2', Journal of Molecular Biology, vol. 373, no. 3, pp. 719-731.

\section{Structure of the Janus Protein Human CLIC2}

Brett A. Cromer, ${ }^{1,8}$ Michael A. Gorman, ${ }^{1,8}$ Guido Hansen, ${ }^{1,8}$ Julian J. Adams, ${ }^{1}$ Marjorie Coggan, ${ }^{2}$ Dene R. Littler, ${ }^{3,4}$ Louise J. Brown, ${ }^{3,4.5}$ Michele Mazzanti, ${ }^{6}$ Samuel N. Breit, ${ }^{4}$ Paul M.G. Curmi, ${ }^{3,4}$ Angela F. Dulhunty, ${ }^{2}$ Philip G. Board ${ }^{2 *}$ and Michael W. Parker ${ }^{1,7^{*}}$

${ }^{1}$ Biota Structural Biology Laboratory, St. Vincent's Institute, 9 Princes Street, Fitzroy, Victoria 3065, Australia

${ }^{2}$ John Curtin School of Medical Research, Australian National University, PO Box 334, Canberra, ACT 2601, Australia

${ }^{3}$ School of Physics, University of New South Wales, NSW 2052, Australia

${ }^{4}$ Centre for Immunology, St. Vincent's Hospital and University of New South Wales, Sydney, NSW 2109, Australia

${ }^{5}$ Department of Chemistry and Biomolecular Sciences, Macquarie University, Sydney, NSW 2109, Australia

${ }^{6}$ Department of Biomolecular Science and Biotechnology, University of Milan, Via Celoria, 26, 20133 Milan, Italy

${ }^{7}$ Department of Biochemistry and Molecular Biology, Bio21 Molecular Science and Biotechnology Institute, The University of Melbourne, 30 Flemington Road, Parkville,

Victoria 3010, Australia

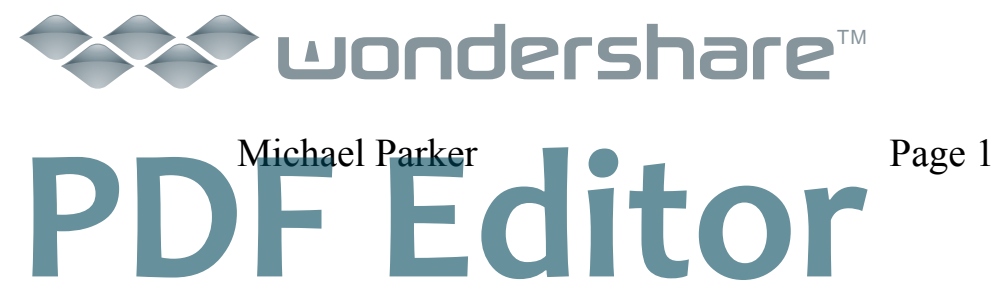


Present addresses: Brett A. Cromer, Howard Florey Institute, University of Melbourne, Victoria 3010, Australia. Julian J. Adams, Australian Synchrotron Project, 800 Blackburn Road, Clayton, Victoria 3168, Australia. Dene R. Littler, The Netherlands Cancer Institute, Division of Molecular Carcinogenesis, Plesmanlaan 121, 1066 CX, Amsterdam, The Netherlands

${ }^{8}$ These are joint first authors.

*Corresponding authors: E-mail addresses: philip.board@anu.edu.au or mparker@svi.edu.au

Running Title: Human CLIC2 structure

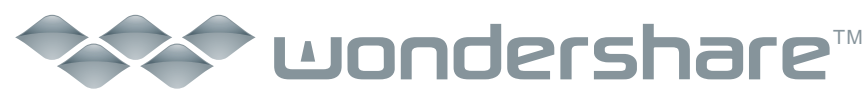




\section{Summary}

Chloride Intracellular Channel (CLIC) proteins possess the remarkable property of being able to convert from a water-soluble to a membrane channel state. We have determined the threedimensional structure of human CLIC2 in its water-soluble form by X-ray crystallography at $1.8 \AA$ resolution from two different crystal forms. In contrast to the previously characterized CLIC1 protein, which forms a possibly functionally important disulfide-induced dimer under oxidizing conditions, we show that CLIC2 possesses an intramolecular disulfide and that the protein remains monomeric irrespective of redox conditions. Site-directed mutagenesis studies show that removal of the intramolecular disulfide or introduction of cysteine residues in CLIC2, equivalent to those that form the intermolecular disulfide in CLIC1, does not cause dimer formation under oxidizing conditions. We show that CLIC2 forms pH-dependent chloride channels in vitro with a $\mathrm{pH}$ optimum of about 5 and the channels are subject to redox regulation. In both crystal forms we observe an extended loop region from the C-terminal domain, called the foot loop, inserting itself into an interdomain crevice of a neighbouring molecule. The equivalent region in the structurally related glutathione transferase superfamily corresponds to the active site. This so-called "foot-in-mouth" interaction suggests that CLIC2 might recognise other proteins such as the ryanodine receptor through this interaction.

Keywords: chloride intracellular channels; CLIC2; pore-forming protein; ryanodine receptor; X-ray crystallography

Abbreviations used: CLICs, chloride intracellular channels; GSH, glutathione; GSTs, glutathione S-transferases; RyR2, cardiac ryanodine receptor 2; SEC, size-exclusion chromatography.

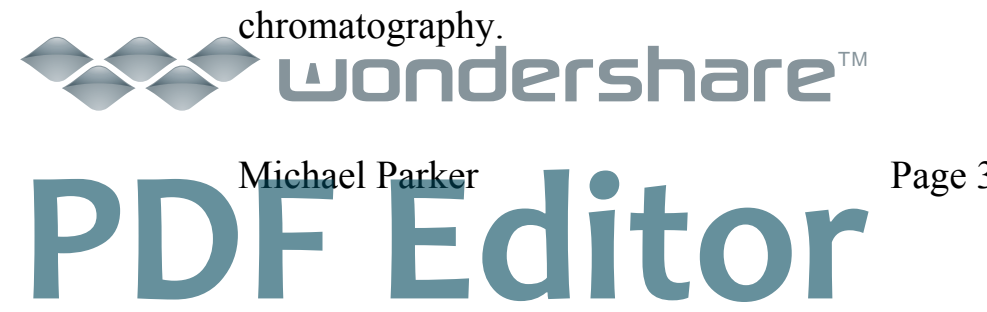


The CLIC (Chloride Intracellular Channel) proteins are a family of highly homologous proteins that display both broad tissue and cellular distribution. They have been implicated in kidney function, ${ }^{1}$ cell division ${ }^{2}$ and bone resorption. ${ }^{3}$ The founding CLIC family member, p64, was discovered in a search for novel chloride channel inhibitors and was characterized as an intracellular chloride channel. ${ }^{4}$ Since then members of the family have been identified in many vertebrates such as amphibians, birds, fish and mammals. They may also exist in invertebrates such as sea squirts, nematodes and insects. ${ }^{1,5}$ Six CLICs have been identified in humans (CLICs 1 to 6$)$, each consisting of a highly conserved core $(40-80 \%$ sequence identity) of $\sim 230$ amino acids with a less homologous hydrophilic N-terminal extension in some. It was proposed that the conserved core would likely adopt the canonical fold of the glutathione S-transferase (GST) superfamily based on sequence similarities ${ }^{6}$ and this was subsequently confirmed with the crystal structure determination of human CLIC1 at $1.4 \AA$ A resolution. ${ }^{7}$

The function of CLIC proteins is not clear. The first CLIC to be characterized, p64 (a CLIC5 homologue), was shown to form chloride channels in planar lipid bilayers and lipid vesicles. ${ }^{8}$ Evidence for other CLIC proteins forming channels has been presented including CLIC1, ${ }^{7,9-11}$ CLIC3, ${ }^{12}$ CLIC4 ${ }^{13}$ and CLIC5 ${ }^{14}$ CLIC proteins can localize to distinct cellular membranes including the nuclear membrane, lysosomal membranes, mitochondria, Golgi membranes, cell-cell junctions and the plasma membrane. ${ }^{15-18}$ CLIC channel activity has been linked to a number of disease processes. Functional expression of CLIC1 chloride channel conductance is associated with microglia-mediated neurotoxicity of $A \beta$, the peptide centrally implicated in Alzheimer's disease. ${ }^{19}$ Altered expression of CLIC4 induces apoptosis in several cell types in vitro and retards growth of grafted tumours in vivo. ${ }^{20}$ However, to date most of the evidence that CLIC proteins form channels is based on in vitro experimental systems and thus theirchysiological ${ }^{\top}$ roles remain to be established. CLICs are known to 
associate with a number of different types of proteins including cytoskeletal and scaffold proteins $^{18,21-23}$ and are the targets of phosphorylation. ${ }^{12,24,25}$ Thus CLIC proteins may play a variety of roles that involve interaction with other proteins or membranes.

The ability of some CLIC proteins to form chloride-selective channels in vitro despite possessing no obvious transmembrane regions of primary structure has made them a fascinating protein family to study. The ability to convert from soluble protein to membrane channel has also been observed for bacterial pore-forming protein toxins ${ }^{26,27}$ and because of this property they are sometimes referred to as Janus proteins after the Roman two-faced god. A class of such toxins called the $\beta$-PFTs do not possess hydrophobic stretches of primary structure but adopt transmembrane regions through dramatic changes in protein structure. Thus there may be common features between CLICs and pore-forming toxins in their mechanism of membrane insertion.

CLIC2 is one of the least characterized CLIC family members. At least two isoforms are known to exist with the difference being an 18 residue insert occurring immediately after the first $\beta$-strand. ${ }^{5}$ CLIC2 is found in most tissues except the brain with high expression levels in the lung and spleen. ${ }^{28}$ CLIC2 inhibits cardiac ryanodine receptor calcium release channels suggesting CLIC2 may function to regulate calcium release from intracellular stores in heart and skeletal muscle. ${ }^{28,29}$

We have initiated structural studies of CLIC2 with the aims of understanding how it forms channels in membranes and whether the structure provides clues to other roles the protein might play. Here we present the structure of human CLIC2 to high resolution determined from two different crystal forms. We show that the protein can form chloride channels in vitro at low $\mathrm{pH}$ that are redox controlled. The crystal structures suggest how CLIC2 can recognise other proteins such as its known interaction with the ryanodine receptor.

\section{4andershare}




\section{Overall structure of human CLIC2}

We have determined the structure of human CLIC2 from two different crystal forms, denoted $\mathrm{A}$ and $\mathrm{B}$, to a resolution of $1.8 \AA$ (Table 1$)$. The CLIC2 molecule is box-shaped $(\sim 60$ $\AA$ x $60 \AA$ x $35 \AA$ ), consisting of two domains (Figure 1(a),(b)). The N-terminal domain (residues 1 to 94) adopts a thioredoxin-like fold consisting of a four stranded mixed $\beta$-sheet with two $\alpha$-helices running parallel to the sheet on one face $(\alpha 1$ and $\alpha 3)$ and one helix $(\alpha 2)$ running perpendicular to the sheet on the other face. The electron density for helix $\alpha 2$ is poorly resolved in both crystal forms where no unambiguous electron density could be obtained for residues 56-65 or residues 61-68 in crystal form A and B, respectively. The Cterminal domain (residues 107 to 245) is all helical and contains a long loop (residues 152 to 180) between helices 5 and 6 , a feature characteristic of the CLIC family which has been referred to previously as the foot loop. ${ }^{7}$ The two domains are connected by an interdomain loop (residues 95 to 106) that is rich in proline residues (4 out of 12 residues). There are two cis prolines in the structure: at the di-proline, Pro 70 - Pro 71, in the N-terminal domain and at another di-proline, Pro 96 - Pro 97, located in the interdomain loop.

In crystal form A two glutathione (GSH) molecules are bound. The first GSH (GSH1) is located on the surface of the N-terminal domain (Figure 1). The second GSH (GSH2) is located between two CLIC molecules in the crystal lattice with contributions from helix $\alpha 5$ of one molecule and the interdomain loop in the other. Both molecules are highly mobile compared to the protein (Table 1). GSH1 binds to a shallow, mostly hydrophobic cavity. The binding site is formed by strand $\beta 2$, residues $24-28$ in loop $\beta 1 / \alpha 1$ and helix $\alpha 1$ in the $N$ terminal domain and helix $\alpha 8$ in the C-terminal domain. The interaction is governed by shape complementarity with two van der Waals contacts and hydrogen bonding (3.1 $\AA$ ) between $\beta 1 / \alpha 1$ loop residue Glu 25 and the amino group of the $\gamma$-glutamyl moiety of GSH. GSH2 binds to a hydrophobic pocketbetween the two symmetry related molecules. The interaction 
is governed by shape complementarity with 3 van der Waals contacts (between Ala 131 and Phe 135 of one molecule and Pro 100 from the other molecule) and hydrogen bonding (3.0 ̊) between Asn 134 and the carboxyl group of the $\gamma$-glutamyl moiety of GSH. GSH was found to be an essential ingredient in the crystallization of both crystal forms although no GSH molecule was identified in form B. There is no evidence of covalent modification by GSH of any of the four cysteine residues in CLIC2.

There is one molecule in the asymmetric unit of each crystal form. They superimpose reasonably well with a root-mean-square deviation of $1.0 \AA$ or $0.7 \AA$ if the foot region is excluded (Figure 1(c)). The largest deviations in the N-terminal domain are: a disordered region between residues 55 and 69 (which includes helix $\alpha 2$ and its flanking regions) and a loop (residues 80 to 84 ) that connects $\beta$-strand 4 to helix $\alpha 3$. The structure of both crystal forms reveal an intramolecular disulfide bridge between Cys 30 and Cys 33 with a right handed hook conformation (Figure 1(d)). The residues in the vicinity of the disulfide superimpose closely between the two crystal forms with the exception of two loops (maximal deviations at Pro 70 and Phe 83 respectively); the changes appear to be due to structural changes transmitted from different conformations of the neighbouring helix $\alpha 4$ (Figure 1 (d) and see below).

Within the C-terminal domain, residues in parts of helix $\alpha 4$ in the $\mathrm{C}$-terminal domain show large deviations in the two crystal forms. In crystal form B the interdomain loop leads to a long, continuous helix $\alpha 4$ (residues 108-125), whereas in form A this helix is broken between residues 112-115 (helix $\alpha 4 \mathrm{a}$, residues 108-111; helix $\alpha 4 \mathrm{~b}$, residues $116-125$ ). Other differences in the C-terminal domain include residue 127 in the loop connecting helices 4 and 5, in the foot loop (residues 154 to 168) and the C-terminus (residues 244 to 245). All the above differences can be ascribed to crystal lattice contacts. The foot loop extends away from helix $\alpha 5$ before making a $180^{\circ}$ turn between residues 158 to 164 (Figure 1(a),(b)). The loop 
then extends perpendicular to helix $\alpha 5$, along the base of helices $\alpha 4$ and $\alpha 7$ before joining helix $\alpha 6$. Taking the foot loop in isolation, both crystal forms display similar topology having root-mean-square deviation of $1.7 \AA$ (Figure $1(\mathrm{c})$ ). This similar topology is due to the maintenance of side-chain to side-chain, side-chain to main-chain and hydrophobic interactions between the two crystal forms and their respective symmetry mates. The differences between the crystals forms within the foot loop are due to slight differences in crystal contacts and limited hydrogen bonding either within the molecules or between symmetry related molecules. $\beta$-strand hydrogen bonding is seen between Ala 163 and Ile 158 in crystal form B (Figure 1(b)) but not in form A. When the structures derived from the two crystals forms are compared in Ramachandran space, the largest deviations are found in the foot loop, namely Ala 163 and Glu 164. The foot loop extends into a groove of a symmetry related molecule that we call the mouth region (Figures 1,2). Out of the 20 residues within the foot loop, seven are negatively charged and fit into the positively charged groove (Figure 2). Hydrophobic interactions are supplied by the highly conserved Ile 158 that sits in a hydrophobic pocket surrounded by residues Val 242, Tyr 239 and the akyl chain of Lys 125 (Figure 2(b)). The root-mean-square deviation between the two crystal forms in the portion of the foot loop that interacts with the mouth region (residues $151-171$ ) is $1.5 \AA$, highlighting the very similar conformations despite the different crystal lattices.

\section{Similarity to other CLIC structures}

The structures of two other CLIC proteins have been determined: human CLIC1 ${ }^{7,11}$ and human CLIC4. ${ }^{30,31}$ CLIC2 shares $60 \%$ sequence identity with CLIC1 and 63\% sequence identity with CLIC4. The overall structures are similar with an overall r.m.s deviation between CLIC2 and CLIC1 (PDB code: $\underline{\mathbf{1 K 0 M}}$ ) on $\alpha$-carbon atoms of $1.8 \AA$ (for 224 matching residues) or $0.9 \AA$ if the foot loop region is excluded (Figure 1(e)). The overall r.m.s 


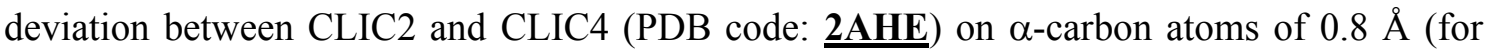
211 matching residues with the foot loop region being disordered in CLIC4) (Figure 1(e)). The most significant differences are at the $\mathrm{N}$ - and $\mathrm{C}$-termini, residues 53 to 70 corresponding to helix $\alpha 2$ and its flanking regions (CLIC1: residues 47-64; CLIC4: residues 58-75) and the foot loop (Figure 1(e)). Helix $\alpha 2$ is highly flexible in other CLIC proteins, being partially disordered in $\mathrm{CLIC}^{31}$ or adopting alternative conformations when crystallised in different crystal forms of $\mathrm{CLIC1}^{7}$ and CLIC4. ${ }^{30,31}$ The foot loop region is highly mobile and even disordered in the CLIC4 structures. Comparing the foot loop region between CLIC2 and CLIC1 (Figure 1(e)), the conformations are similar for the first three residues to residue Leu 154 (Leu 148 in CLIC1). This highly conserved residue lies in a hydrophobic pocket between helices $\alpha 4$ and $\alpha 5$. In CLIC2, the foot loop then adopts a hairpin turn due to a di-proline sequence which instead is a short helix in CLIC1. The loop regions converge again at the highly conserved residue Arg 171 (Arg 165 in CLIC1). This residue makes both side-chain and main-chain interactions with residues located in helix $\alpha 5$. The foot loop in CLIC4 appears to protrude into the mouth region of a neighbouring molecule although a lot of the region is disordered in the crystal. ${ }^{30}$ In crystal form B helix 4 forms a continuous helix whereas in all other CLIC structures this helix is broken between residues 112 and 115 .

In the CLIC1 structure GSH was found bound to Cys 24 (equivalent to Cys 30 in CLIC2), which is located in the interdomain interface and corresponds to the GSH binding site in GSTs (see below) (Figure 1(f)). ${ }^{7}$ Many of the interactions between GSH and CLIC1 were retained by analogous residues in the GSTs. ${ }^{32}$ However, most of the residues in CLIC1 that interact with GSH are lost in CLIC2 with the exceptions of Lys 19 (Lys 13 in CLIC1) which could interact with the GSH glycyl carboxylate and Cys 30 (Cys 35 in CLIC1) which could form a mixed disulfide with GSH (Figure 1(f)). The introduction of the diproline at residues70-71 disrupts the XXX-cis-Pro motif seen in CLIC1 and GSTs which forms an anti- 
parallel $\beta$-sheet interaction with the backbone of GSH. This diproline motif is conserved in all vertebrate CLIC2, CLIC4, CLIC5 and CLIC6 proteins while the Leu-Pro variant is only seen in mammalian CLIC1 and mammalian and avian CLIC3. ${ }^{5}$ In CLIC2 Phe 83 would block the $\gamma$-glutamyl portion of GSH from binding (Figure 1(f)). Finally, the disulfide between Cys 30 and Cys 33 of CLIC2 would need to be reduced to allow GSH to bind (Figure 1(f)). In summary, the GSH binding site seen in CLIC1 is absent in CLIC2.

\section{Similarity to GSTs}

CLIC2 adopts the same topology of secondary structure as GSTs and most closely resembles beta, omega, tau and zeta GSTs with r.m.s. deviations on $\alpha$-carbon atoms of approximately $2 \AA$ against each GST. This value is well within the range found for superposition of GSTs from different classes. ${ }^{32}$ Four key residues are conserved in virtually all GSTs and CLICs: a cis-proline residue (Pro 71 in CLIC2) that provides the correct active site geometry for binding GSH in GSTs, an aspartic acid residue (Asp 183 in CLIC2) that forms an N-terminal helix capping interaction and two glycine residues that play structural roles. An obvious difference between CLIC2 and GSTs is that the former is a monomer whereas the latter are dimers. However, there are at least two reported cases of monomeric GSTs $^{33,34}$ and in the beta ${ }^{35}$ and sigma ${ }^{36}$ class GSTs the dimer interface is very polar which is thought to be a relic of their ancestral origins within the monomeric thioredoxin superfamily. The CLIC2 domain interface is predominantly hydrophobic and the linker region between the two domains provides a large hydrophobic residue (Leu 102 in CLIC2) as a wedge between the two domains as found in most GST structures.

There is some evidence that CLIC2 possesses enzymatic activity ${ }^{28}$ and it is likely that its active site (that we term the mouth region) is in the same location as found in GSTs. The GST active site is located at a erevice between the two domains and consists of a GSH 
binding site (G-site) and a binding site for hydrophobic electrophilic compounds (the H-site). A characteristic feature of GSTs is the mode of GSH binding: GSH binds in an extended fashion so that it forms an anti-parallel $\beta$-sheet-like interaction between its peptide backbone and the backbone of the residue preceding the strictly conserved cis-proline residue in the protein. We don't observe GSH binding in the mouth region of either crystal form despite the presence of GSH in the crystallisation conditions. GST residues involved in binding GSH are nearly all substituted by non-conservative replacements in CLIC2 and hence there is no obvious GSH binding site in CLIC2 (also see above). Helix $\alpha 2$, which lines the G-site, is normally a highly flexible region in mammalian GSTs as it is in both crystal forms of CLIC2 reported here. The most striking feature of the CLIC2 mouth region is the presence of the intramolecular disulfide bridge between Cys 30 and Cys 33 (Figure 1(b)).

The partly buried H-site in CLIC2 is reminiscent of the human alpha ${ }^{37}$ and theta class $^{38}$ structures where the active sites are also partly covered by a C-terminal helical extension. Nevertheless, the CLIC2 mouth region is located in a wide crevice (Figure 2) which is reminiscent of some GST active sites, particularly the beta and omega class GSTs where it has been suggested that the site is sufficiently large to accept protein targets. ${ }^{35,39}$

An obvious difference between the CLIC and GST families is the long, highly charged loop region, the foot loop, (from Thr 152 to Thr 180) at the base of the CLIC molecule. In CLIC2 there are 8 acidic residues in this region and the number varies between 3 and 7 in other family members. Another hotspot of negative charge occurs in the C-terminal helix $\alpha 9$ (residues 232 to 239) where there are 3 acidic residues. CLIC2 has a pronounced basic mouth region like many GSTs (Figure 2).

\section{$\$$ uondershare ${ }^{\text {Tu }}$




\section{Influence of the intramolecular disulfide on oligomer formation}

The structure of CLIC2 revealed an intramolecular disulfide bridge between Cys 30 and Cys 33 (Figure 1(d)). In contrast, CLIC1 was shown to have no disulfides in the monomer state but an intramolecular disulfide formed in the dimer state between residues Cys 24 and Cys 59. ${ }^{11}$ The corresponding residues in CLIC2 are Cys 30 and Ala 65 and hence such a disulfide is not possible in CLIC2. Nevertheless we wanted to examine whether CLIC2 could undergo the same dramatic changes in conformation by engineering cysteine residues at the appropriate positions. The following mutants were generated: (1) C33A in order to remove the intramolecular disulfide in CLIC2, (2) A65C to make the equivalent disulfide (Cys 30/Cys 65) observed in the CLIC1 dimer possible, and (3) C33A/A65C in order to stabilise the possible Cys 30/Cys 65 disulfide by eliminating both the Cys 30/Cys 33 disulfide and the possibility of a Cys 33/Cys 65 disulfide.

We used size-exclusion chromatography (SEC) and SDS-PAGE to test the effect of these mutations on the oligomeric state of CLIC2 under varying redox conditions (data not shown). Wild-type CLIC2 runs as a monomer as judged by SEC under both reducing and oxidising conditions and under different ionic strength conditions. The C33A mutant runs as a monomer under reducing conditions but with evidence of a small amount of dimer and larger amounts of higher aggregate formation under oxidising conditions. The latter do not appear to be linked by intermolecular disulfides as judged by SDS-PAGE. The A65C mutant is also monomeric under reducing conditions but increasing levels of dimers are observed under oxidising conditions. SDS-PAGE reveals that these dimers contain intermolecular disulfides so are not analogous to $\mathrm{CLIC1}$ dimers. The double mutant $\mathrm{C} 33 \mathrm{~A} / \mathrm{A} 65 \mathrm{C}$ runs mostly as a monomer under reducing conditions with some small amounts of dimer and higher order aggregates. The amount of dimer and higher order aggregates increases only slightly under oxidising conditions, indicating some involvement of C33 in the disulfide-linked dimers seen uondershare ${ }^{\text {TM }}$ 
for the $\mathrm{A} 65 \mathrm{C}$ mutant. In summary, the mutations do not provide any evidence that CLIC2 can undergo a conformational change and dimerisation similar to that seen for a CLIC1.

\section{Chloride efflux experiments}

Previous studies have shown that both CLIC1 and CLIC4 produce chloride efflux from artificial liposomes with the flux increasing at acidic $\mathrm{pH} .{ }^{10,11,30}$ The flux data for human CLIC2 appears to be very similar to what was observed previously for CLIC1 and CLIC4, ${ }^{11,30}$ except with a small notable $\mathrm{pH}$ shift in its response (Figure 3(a)). The CLIC2 flux is minimal at $\mathrm{pH} 8$ and increases markedly with decreasing $\mathrm{pH}$. The other CLIC proteins differ in the position of the efflux minimum, which occurs between $\mathrm{pH} 7$ to 7.5 for $\mathrm{CLIC}^{30}$ and $\mathrm{pH} 7.0$ for CLIC1. ${ }^{10}$

\section{Channel activity}

Single channel currents were observed when soluble CLIC2 was added to artificial lipid bilayers using tip dip electrophysiology (Figure 3(b)). The single channel conductance was measured at $48.4 \pm 1.2 \mathrm{pS}$, which is higher than the conductance observed for CLIC1 $(28 \pm 9 \mathrm{pS})^{11,40}$ and CLIC4 $(30 \pm 2 \mathrm{pS})^{30}$ under similar conditions (Figure 3(c)). The probability of observing single channel currents after the addition of CLIC2 to the tip dip chamber shows a strong $\mathrm{pH}$ dependence, with no currents observed at $\mathrm{pH} 8$, increasing to 0.67 at $\mathrm{pH} 5$ (Figure 3(d)). This is consistent with the $\mathrm{pH}$ response observed in the chloride efflux experiments with a minimum at $\mathrm{pH} 8.0$ (Figure 3(a)). When current measurements were carried out under reducing conditions, in the presence of $5 \mathrm{mM}$ DTT, no channel activity was observed, as seen for CLIC1 ${ }^{11}$ and CLIC4. ${ }^{30}$ Channel opening sub-states were observed for CLIC2 (data

\section{2 uondershare}


not shown). Similar sub-states have been observed for other CLICs and interpreted as CLIC channels consisting of variable number of subunits. ${ }^{40,41}$

\section{Functional consequences of similarities to other proteins?}

A search of the Protein Data Bank for similar folds to CLIC2 shows it most closely resembles the beta, omega, tau and theta classes of GSTs (after omitting hits to other CLIC proteins). Despite the structural similarities between GSTs and CLICs, CLIC2 has been shown to display a low affinity for GSH and is not retained on GSH affinity columns. ${ }^{28}$ Nor do we see GSH bound to the mouth region in either crystal form despite it being an essential ingredient in the crystallization of both forms. Furthermore, nearly all the GST residues that are normally found to interact with GSH are replaced by non-conservative substitutions in CLIC2 to such an extent that the site no longer looks like it is capable of binding GSH. In crystal form A, we do see a GSH molecule bound weakly to a surface of the N-terminal domain (Figure 1(a)) but the paucity of interactions between protein and ligand suggests the observed interaction is unlikely to be physiologically relevant. In contrast GSH does bind to the mouth region of CLIC1 where it forms a mixed disulfide bond between it and Cys 24 (Figure 1(f)), a highly conserved residue in the CLIC protein family, ${ }^{7}$ which corresponds to the catalytically essential cysteine in omega class GSTs. ${ }^{39}$ However, even with CLIC1, no non-covalent GSH binding was observed and in the crystal structure of the covalent GSHCLIC1 complex, the GSH moiety shows considerable disorder with few specific non-covalent interactions with the protein. ${ }^{7}$

The beta and omega GSTs, like CLIC $2,{ }^{28}$ do not display GSH-conjugating activity towards a broad range of common GST substrates which has prompted searches for novel functions. ${ }^{35,39,42,43}$ All three proteins possess a conserved cysteine residue located at the Nterminus of helix al. Sth the omega ${ }^{\mathrm{m}}$ GSTs and CLICs the cysteine residue is part of a 
sequence motif, $\mathrm{CP}[\mathrm{FS}][\mathrm{CSA}]$, which corresponds to the $\mathrm{CXX}[\mathrm{CS}]$ active site motif of the thioredoxin superfamily. In CLIC2 both cysteines of the motif are present, unlike in CLIC1 and omega class GSTs where only the first cysteine is conserved. In oxidised forms of thioredoxin family members, the two cysteines can form an intramolecular disulfide as seen in CLIC2. This raises the possibility that CLIC2 could have glutaredoxin-type activity. However, CLIC2 displays no thioltransferase activity making it distinct from glutaredoxins and omega class GSTs. ${ }^{28}$ 3-D fold similarity searches revealed that CLIC2 closely resembles a number of thioredoxin superfamily members including $E$. coli glutaredoxin 2 (Grx2; PDB code: 1970) and its N-terminal domain resembled NrdH-redoxin (PDB code: $\underline{\mathbf{1 h 7 5}}$ ) and a mammalian thioltransferase (PDB code: $\underline{1 \text { kte)}}$. Of particular interest is NRDH-redoxin which, like CLIC2, possesses a CXXC motif where the cysteines are disulfide bonded, and does not have a GSH binding site despite possessing significant homology to glutaredoxins but has a wide H-site suggesting it could interact with redox protein partners. ${ }^{44}$ CLIC2 does display weak peroxidase activity, an activity it shares with many GST family members. ${ }^{28}$ These observations suggest that CLIC2 may have some yet to be discovered enzymatic activity.

\section{CLIC2 recognition of binding partners}

The insertion of the foot loop into the CLIC2 mouth region of neighbouring molecules, as observed for CLIC4, ${ }^{30}$ is suggestive that CLIC2 might bind other proteins. By analogy with thioredoxins, Harrop et al. ${ }^{7}$ have previously postulated that the wide mouth region of CLICs might accommodate a putative protein target. Within the CLIC family there is a relatively low sequence conservation of the foot loop although it does tend to possess a net negative charge. The conformations of the foot loop regions that enter the mouth regions of neighbouring CLIC2 molecules in our structures are strikingly similar in both crystal forms

(Figure 1(c)). The mouth region itself bears a net positive charge and hence complements the

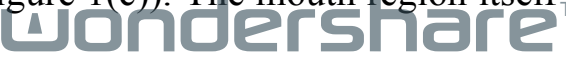


net negative charge of the foot loop (Figure 2). The region of interaction is from residue Asp 156 to Pro 167 of the foot loop with a mixture of main-chain and side-chain interactions.

CLIC2 is a known regulator of cardiac ryanodine receptor 2 (RyR2) channels and does so by binding directly to the cytoplasmic side of the channels. ${ }^{29}$ Mutation of Cys 30 in CLIC2 markedly affects its inhibitory properties on RyR2 channels suggesting the receptor binds to the mouth region of CLIC $2 .{ }^{29}$ Based on our observations of the acidic foot loop region binding into the mouth of CLIC molecules, we searched the amino acid sequence of RyR2 for acidic-rich stretches. We found 25 such stretches (defined as 5 or more acidic resides in 12 residues windows). One stretch, 1988-DDKSECPCPEE-1998, looks particularly intriguing with two cysteines and two proline residues. The CLIC2 mouth region is rich in cysteine and proline residues (Figure 1(f)) and the acidic rich loop that pokes into the mouth has proline residues near its tip. This region in RyR2 is located in the cytoplasmic domain of the channel $^{45}$ consistent with the observation that CLIC2 binds to this domain. ${ }^{29}$

\section{Concluding remarks}

In this present study we have determined the crystal structure of human CLIC2. We show it adopts a very similar fold to the CLIC1 and CLIC4 proteins with the major differences being in the mobile helix $\alpha 2$ region and the foot loops. Two unusual features of the CLIC2 structure are the presence of an intramolecular disulfide in the N-terminal domain and the insertion of the foot loop regions into the mouth regions of neighbouring molecules in the crystal lattice. We demonstrate for the first time that CLIC2 can form chloride channels with maximal activity at low $\mathrm{pH}$ and that channel activity is controlled by redox conditions with activity disappearing in the presence of reducing agent.

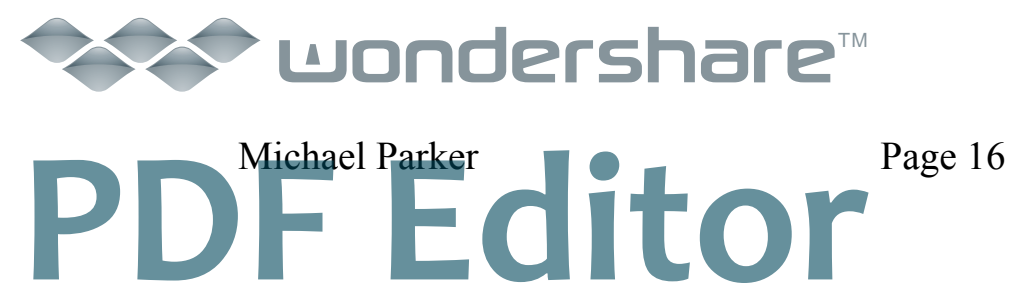


A number of workers have speculated how CLIC proteins can insert into membranes to form ion channels $s^{7,11,32,41,46}$ but there is a paucity of supporting experimental data. We will use the CLIC2 crystal structure to guide for further experimental work with the aim of testing the various models of membrane insertion. 


\section{Protein Data Bank accession numbers}

The atomic coordinates and structure factors (accession codes XXX and YYY) have been deposited in the Protein Data Bank, Research Collaboratory for Structural Bioinformatics, Rutgers University, New Brunswick, NJ (http://www.rcsb.org).

\section{Acknowledgments}

We thank Dr Yibin Xu for advice. We also thank Harry Tong and other staff at BioCARS for their help with data collection during our visit to the Advanced Photon Source. This work, including use of the BioCARS sector, was supported by the Australian Synchrotron Research Program, which is funded by the Commonwealth of Australia under the Major National Research Facilities Program. Use of the Advanced Photon Source was supported by the U.S. Department of Energy, Basic Energy Sciences, Office of Energy Research. This work was also supported by grants from the National Health and Medical Research Council of Australia (NHMRC) and Australian Research Council (ARC) to P.G.B., A.F.D., P.M.G.C., B.A.C. and M.W.P. M.W.P. is an ARC Federation Fellow and NHMRC Honorary Fellow.

\section{2 undershare ${ }^{\text {Tw }}$}




\section{References}

1. Berry, K.L., Bulow, H.E., Hall, D.H. \& Hobert, O. (2003) A C. elegans CLIC-like protein required for intracellular tube formation and maintenance. Science 302, 21342137.

2. Valenzuela, S.M., Mazzanti, M., Tonini, R., Qui, M.R., Warton, K., Musgrove, E.A., Campbell, T.J. \& Breit, S.N. (2000). The nuclear chloride ion channel NCC27 is involved in regulation of the cell cycle. J. Physiol. (Lond.) 529, 541-552.

3. Schlesinger, P.H., Blair, H.C., Teitelbaum, S.L. \& Edwards, J.C. (1997). Characterization of the osteoclast ruffled border chloride channel and its role in bone resorption. J. Biol. Chem. 272, 18636-18643.

4. Landry, D., Sullivan, S., Nicolaides, M., Redhead, C., Edelman, A., Field, M., alAwqati, Q., \& Edwards, J. (1993). Molecular cloning and characterization of p64, a chloride channel from kidney microsomes. J. Biol. Chem. 268, 14948-14955.

5. Littler, D.R. (2005). Structural studies of CLIC proteins. PhD thesis, University of New South Wales, Australia.

6. Dulhunty, A., Gage, P., Curtis, S., Chelvanayagam, G. \& Board, P.G. (2001). The glutathione transferase structural family includes a nuclear chloride channel and a ryanodine receptor calcium release channel modulator. J. Biol. Chem. 276, 3319-3323.

7. Harrop, S.J., DeMaere, M.Z., Fairlie, W.D., Reztsova, T., Valenzuela, S.M., Mazzanti, M., Tonini, R., Qui, M.R., Jankova, L., Warton, K., Bauskin, A.R., Wu, W.M., Pankhurst, S., Campbell, T.J., Breit, S.N. \& Curmi, P.M.G. (2001). Crystal structure of the soluble form of the intracellular chloride ion channel CLIC1 (NCC27) at $1.4 \AA$ resolution. J. Biol. Chem. 276, 44993-45000.

8. Edwards, J.C. (1999). A novel p64-related $\mathrm{Cl}^{-}$channel: subcellular distribution and nephron segment-specific expression. Am. J. Physiol. 276, F398-F408. 
9. Tulk, B.M., Schlesinger, P.H., Kapadia, S.A. \& Edwards, J.C. (2000). CLIC-1 functions as a chloride channel when expressed and purified from bacteria. J. Biol. Chem. 275, 26986-26993.

10. Tulk, B.M., Kapadia, S. \& Edwards, J.C. (2002). CLIC1 inserts from the aqueous phase into phospholipids membranes where it functions as an anion channel. Am. J. Physiol. 282, C1103-C1112.

11. Littler, D.R., Harrop, S.J., Fairlie, W.D., Brown, L.J., Pankhurst, G.J., Pankhurst, S., DeMaere, M.Z., Campbell, T.J., Bauskin, A.R., Tonini, R., Mazzanti, M., Breit, S.N. \& Curmi, P.M.G. (2004). The intracellular chloride ion channel protein CLIC1 undergoes a redox-controlled structural transition. J. Biol. Chem. 279, 9298-9305.

12. Qian, Z., Okuhara, D., Abe, M.K. \& Rosner, M.R. (1999). Molecular cloning and characterization of a mitogen-activated protein kinase-associated intracellular chloride channel. J. Biol. Chem. 274, 1621-1627.

13. Proutski, I., Karoulias, N. \& Ashley, R.H. (2002). Overexpressed chloride intracellular channel protein CLIC4 (p64H1) is an essential component of novel plasma membrane anion channels. Biochem. Biophys. Res. Commun. 297, 317-322.

14. Berryman, M., Bruno, J., Price, J. \& Edwards, J.C. (2004). CLIC-5A functions as a chloride channel in vitro and associates with the cortical actin cytoskeleton in vitro and in vivo. J. Biol. Chem. 279, 34794-34801.

15. Valenzuela, S.M., Martin, D.K., Por, S.B., Robbins, J.M., Warton, K., Bootcov, M.R., Scholfield, P.R., Campbell, T.J. \& Breit, S.N. (1997). Molecular cloning and expression of a chloride ion channel of cell nuclei. J. Biol. Chem. 272, 12575-12582.

16. Fernandez-Salas, E., Sagar, M., Cheng, C., Yuspa, S.H. \& Weinberg, W.C. (1999). p53 and tumor necrosis factor $\alpha$ regulate the expression of a mitochondrial chloride channel protein. J. Biol. Chem. 274, 36488-36497. unndershare ${ }^{\text {TM }}$ 
17. Tonini, R., Ferroni, A., Valenzuela, S.M., Warton, K., Campbell, T.J., Breit, S.N. \& Mazzanti M. (2000). Functional characterization of the NCC27 nuclear protein in stable transfected CHO-K1 cells. FASEB J. 14, 1171-1178.

18. Berryman, M.A. \& Goldenring, J.R. (2003). CLIC4 is enriched at cell-cell junctions and colocalizes with AKAP350 at the centrosome and midbody of cultured mammalian cells. Cell Motil. Cytoskeleton 56, 159-172.

19. Novarino, G., Fabrizi, C., Tonini, R., Denti, M.A., Malchiodi-Albedi, F., Lauro, G.M., Sacchetti, B., Paradisi, S., Ferroni, A., Curmi, P.M., Breit, S.N. \& Mazzanti, M. (2004). Involvement of the intracellular ion channel CLIC1 in microglia-mediated $\beta$-amyloidinduced neurotoxicity. J. Neurosci. 24, 5322-5330.

20. Suh, K.S., Mutoh, M., Gerdes, M., Crutchley, J.M., Mutoh, T., Edwards, L.E., Dumont, R.A., Sodha, P., Cheng, C., Glick, A. \& Yuspa, S.H. (2005). Antisense suppression of the chloride intracellular channel family induces apoptosis, enhances tumor necrosis factor $\{$ alpha $\}$-induced apoptosis, and inhibits tumor growth. Cancer Res. 65, 562-571.

21. Suginta, W., Karoulias, N., Aitken, A. \& Ashley, R.H. (2001). Chloride intracellular channel protein CLIC4 (p64H1) binds directly to dynamin I in a complex containing actin, tubulin and 14-3-3 isoforms. Biochem. J. 359, 55-64.

22. Shanks, R.A., Larcocca, M.C., Berryman, M., Edwards, J.C., Urushidani, T., Navarre, J. \& Goldenring, J.R. (2002). AKAP350 at the Golgi apparatus. II. Association of AKAP350 with a novel chloride intracellular channel (CLIC) family member. J. Biol. Chem. 277, 40973-40980.

23. Griffon, N., Jeanneteau, F., Prieur, F., Diaz, J. \& Sokoloff, P. (2003). CLIC6, a member of the intracellular chloride channel family, interacts with dopamine $\mathrm{D}(2)$-like receptors. Brain Res. Mol. Brain Res. 117, 47-57.

\section{unndershare}


24. Duncan, R.R., Westwood, P.K., Boyd, A. \& Ashley, R.H. (1997). Rat brain p64H1: expression of a new member of the p64 chloride channel protein family in endoplasmic reticulum. J. Biol. Chem. 272, 23880-23886.

25. Edwards, J.C. \& Kapadia, S. (2000). Regulation of the bovine kidney microsomal chloride channel p64 by p59fyn, a src family kinase. J. Biol. Chem. 275, 31826-31832.

26. Parker, M.W. \& Feil, S.C. (2005). Pore-forming protein toxins: from structure to function. Prog. Mol. Biol. Biophys. 88, 91-142.

27. Parker, M.W. \& Pattus, F. (1993). A common packaging motif in bacterial protein toxins. Trends Biochem. Soc. 18, 391-395.

28. Board, P.G., Coggan, M., Watson, S., Gage, P.W. \& Dulhunty, A.F. (2004). CLIC-2 modulates cardiac ryanodine receptor $\mathrm{Ca}^{2+}$ release channels. Intl. J. Biochem. Cell Biol. 36, 1599-1612.

29. Dulhunty, A.F., Pouliquin, P., Coggan, M., Gage, P.W. \& Board, P.G. (2005). A recently identified member of the glutathione transferase structural family modifies cardiac RyR2 substate activity, coupled gating and activation by $\mathrm{Ca}^{2+}$ and ATP. Biochem. J. 390, 333-343.

30. Littler, D.R., Assaad, N.N., Harrop, S.J., Brown, L.J., Pankhurst, G.J., Luciani, P., Aguilar, M-I., Mazzanti, M., Berryman, M.A., Breit, S.N. \& Curmi, P.M.G. (2005). Crystal structure of the soluble form of the redox-regulated chloride ion channel protein CLIC4. FEBS J. 272, 4996-5007.

31. Li, Y-F., Li, D-F., Zeng, Z-H \& Wang, D-C. (2006). Trimeric structure of the wild soluble chloride intracellular ion channel CLIC4 observed in crystals. Biochem. Biophys. Res. Commun. 343, 1272-1278.

32. Cromer, B.A., Morton, C.J., Board, P.G. \& Parker, M.W. (2002). From glutathione transferase to pore in a CLIC. Eur. Biophys. J. 31, 356-364.

\section{uondershare ${ }^{\mathrm{T}}$}


33. McCarthy, D.L., Navarrete, S., Willett, W.S., Babbitt, P.C. \& Copley, S.D. (1996). Exploration of the relationship between tetrachlorohydroquinone dehalogenase and the glutathione S-transferase superfamily. Biochemistry 35, 14634-14642.

34. Polekhina, G., Board, P.G., Blackburn, A.C. \& Parker, M.W. (2001). The crystal structure of maleylacetoacetate isomerase/glutathione transferase zeta reveals the molecular basis for its remarkable catalytic promiscuity. Biochemistry 40, 1567-1576.

35. Rossjohn, J., Polekhina, G., Feil, S.C., Allocati, N., Masulli, M., Di Ilio, C. \& Parker, M.W. (1998). A mixed disulfide bond in bacterial glutathione transferase: functional and evolutionary implications. Structure 6, 721-734.

36. Ji, X., van Rosenvinge, E.C., Johnson, W.W., Tomarev, S.I., Piatigorsky, J., Armstrong, R.N. \& Gilliland, G.L. (1995). Three-dimensional structure, catalytic properties, and evolution of a sigma class glutathione transferase from squid, a progenitor of the lens Scrystallins of cephalopods. Biochemistry 34, 5317-5328.

37. Sinning, I., Kleywegt, G.J., Cowan, S.W., Reinemer, P., Dirr, H.W., Huber, R., Gilliland, G.L., Armstrong, R.N., Ji, X., Board, P.G., Olin, B., Mannervik, B. \& Jones, T.A. (1993) Structure determination and refinement of human alpha class glutathione transferase $\mathrm{A} 1-1$, and a comparison with the $\mathrm{Mu}$ and $\mathrm{Pi}$

38. Rossjohn, J., McKinstry, W.J., Oakley, A.J., Verger, D., Flanagan, J., Chelvanayagam, G., Tan, K-L., Board, P.G. \& Parker, M.W. (1998). Human theta class glutathione transferase: the crystal structure reveals a sulfate-binding pocket within a buried active site. Structure 6, 309-322.

39. Board, P.G., Coggan, M., Chelvanayagam, G., Easteal, S., Jermiin, L.S., Schulte, G.K., Danley, D.E., Hoth, L.R., Griffor, M.C., Kamath, A.V., Rosner, M.H., Chrunyk, B.A., Perregaux, D.E., Gabel, C.A., Geoghegan, K.F. \& Pandit, J. (2000). Identification, characterization, and crystal structure of the Omega class glutathione transferases. $J$. Biol. Chem. 275, 24798-24806. 
40. Warton, K., Tonini, R., Fairlie, W.D., Matthews, J.M., Valenzuela, S.M., Qui, M.R., Wu, W.M., Pankhurst, S., Bauskin, A.R., Harrop, S.J., Campbell, T.J., Curmi, P.M., Breit, S.N. \& Mazzanti, M. (2002). Recombinant CLIC1 (NCC27) assembles in lipid bilayers via a $\mathrm{pH}$-dependent two-state process to form chloride ion channels with identical characteristics to those observed in CHO cells expressing CLIC1. J. Biol. Chem. 277, 26003-26011.

41. Singh, H. \& Ashley, R.H. (2006). Redox regulation of CLIC1 by cysteine residues associated with the putative channel pore. Biophys. J. 90, 1628-1638.

42. Laliberte, R. E., Perregaux, D. G., Hoth, L. R., Rosner, P. J., Jordan, C. K., Peese, K. M., Eggler, J. F., Dombroski, M. A., Geoghegan, K. F. \& Gabel, C. A. (2003). Glutathione S-transferase omega 1-1 is a target of cytokine release inhibitory drugs and may be responsible for their effect on interleukin-1beta posttranslational processing. $J$. Biol. Chem. 278, 16567-16578.

43. Board, P.G. \& Anders, M.W. (2007). Glutathione transferase Omega 1 catalyzes the reduction of $S$-(Phenacyl)glutathiones to acetophenones. Chem. Res. Toxicol. 20, 149154.

44. Stehr, M., Schneider, G., Åslund, F., Holmgren, A. \& Lindqvist, Y. (2001). Structural basis for the thioredoxin-like activity profile of the glutaredoxin-like NrdH-redoxin from Escherichia coli. J. Biol. Chem. 276, 35836-35841.

45. Du, G.G., Sandhu, B., Khanna, V.K., Guo, X.H. \& MacLennan, D.H. (2002). Topology of the $\mathrm{Ca}^{2+}$ release channel of skeletal muscle sarcoplasmic reticulum (RyR1). Proc. Natl. Acad. Sci. USA 99, 16725-16730.

46. Berry, K.L. \& Hobert, O. (2006). Mapping functional domains of chloride intracellular channel (CLIC) proteins in vivo. J. Mol. Biol. 359, 1316-1333.

47. DeLano, W. L. (2002). DeLano Scientific, San Carlos, CA, USA. udndershore ${ }^{\mathrm{TM}}$ 
48. Navaza, J. (1994). AMoRe: an Automated Package for Molecular Replacement. Acta Crystallog. Sect A, 50 157-163.

49. Roussel, A. \& Cambillau, C. (198). Silicon Graphics Geometry Partner Directory. Silicon Graphics, Mountain View, CA, 72-78.

50. Brünger, A. T., Adams, P. D., Clore, G. M., DeLano, W. L., Gros, P., GrosseKunstleve, R. W., Jiang, J.-S., Kuszewski, J., Nilges, M., Pannu, N. S., Read, R.G., Rice, R.M. Simonson, T. \& Warren, G.L. (1998). Crystallography and NMR system: A new software suite for macromolecular structure determination. Acta Crystallog. Sect $D, \mathbf{5 4}, 905-921$.

51. Murshudov, G. N., Vagin, A. A. \& Dodson, E. J. (1997). Refinement of macromolecular structures by the maximum-likelihood method. Acta Crystallog. Sect $D, \mathbf{5 3}, 240-255$.

52. Painter, J. \& Merritt, E. A. (2006). Optimal description of a protein structure in terms of multiple groups undergoing TLS motion. Acta Crystallog. Sect D, 62, 439-450.

53. Winn, M. D., Isupov, M. N. \& Murshudov, G. N. (2001). Use of TLS parameters to model anisotropic displacements in macromolecular refinement. Acta Crystallog. Sect $D, \mathbf{5 7}, 122-133$.

54. Laskowski, R. A., MacArthur, M. W., Moss, D. S. and Thornton, J. M. (1993). PROCHECK: a program to check the stereochemical quality of protein structures. $J$. Appl. Crystallogr. 26, 283-291.

55. CCP4 (Collaborative Computational Project) Program Suite (1994). The CCP4 Suite: programs for protein crystallography. Acta Crystallog. Sect D, 50, 760-763.

56. Storoni, L. C., McCoy, A. J. \& Read, R. J. (2004). Likelihood-enhanced fast rotation functions. Acta Crystallog. Sect D, 60, 432-438.

57. Emsley, P. \& Cowtan, K. (2004). Coot: model-building tools for molecular graphics.

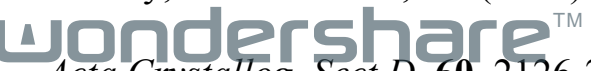
Acta Crystallog. Sect D, 60, 2126-2132. 
58. Catanzariti, A.M., Soboleva, T.A., Jans, D.A., Board, P.G. \& Baker, R.T. (2004). An efficient system for high-level expression and easy purification of authentic recombinant proteins. Protein Sci. 13, 1331-1339. 


\section{Figure legends}

Figure 1. Three-dimensional structure of CLIC2. Views in ribbon style of CLIC2 determined from both crystal forms. (a) Crystal form A with the two bound GSH molecules shown in stick fashion. The foot loop region from a symmetry related molecule is shown in magenta. (b) Crystal form B. The foot loop region from a symmetry related molecule is shown in cyan. (c) An overlay of the two structures shown in ribbon representation and using the same colour scheme as in panels (a) and (b). (d) Close up view of the CLIC2 mouth region showing a superposition of the two crystal forms. The structure from crystal form B is shown in pink. An intramolecular disulfide is formed between Cys 30 and Cys 33 and preserved in both structures. Key residues discussed in the text are shown in stick fashion. (e) Superposition of the alpha-carbon trace of all 12 independent views of available CLIC crystal structures. CLIC2 from crystal forms A and B are shown in blue and brown respectively. The other CLIC structures ${ }^{7,30,31}$ are shown in grey. (f) Superposition of the mouth region of CLIC2 and human CLIC1. ${ }^{7}$ The latter is shown in green. Bound GSH from CLIC1 is shown in yellow stick. Residue labels are for CLIC2 only. All figures were generated using the program PYMOL. ${ }^{47}$

Figure 2. Interaction between foot loop and the mouth region in symmetry related CLIC2 molecules. The surface of the CLIC2 structure (crystal form A) is coloured by residue property: blue is basic and red is acidic. The foot loops of symmetry related CLIC2 molecules (residues 156 to 164) are coloured in magenta and green for crystal forms A and B, respectively with main-chain atoms shown as a "worm" and side-chain atoms as sticks. (a) Orthogonal views of the foot loop binding into a deep, positively charged groove. (b) Close up view into the foot loop interaction site.

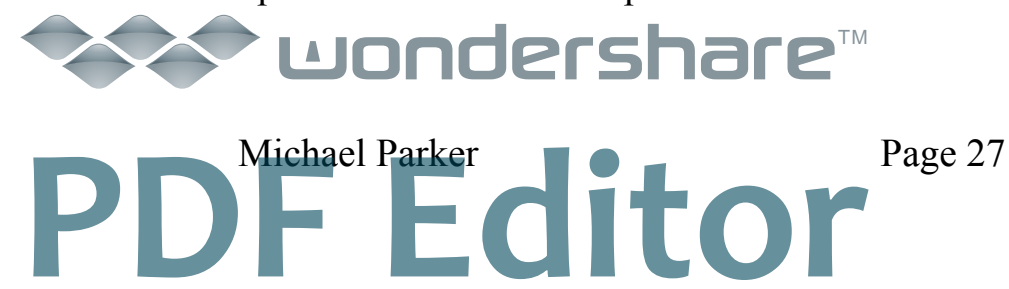


Figure 3. Chloride efflux and tip-dip experiments on human CLIC2. (a) The pH effect on the chloride efflux for human CLIC2. The data shown are representative of two independent experiments over the $\mathrm{pH}$ range of 5.0 to 9.0. CLIC2 protein (grey bars) or control buffer (black bars) were added to the chloride loaded liposomes in the required chloride-free $\mathrm{pH}$ buffer. $1.0 \mu \mathrm{M}$ valinomycin was added to potentiate the chloride-driven efflux from the liposomes and the chloride release monitored continuously for 240 seconds. Triton X-100 $(1 \% \mathrm{v} / \mathrm{v})$ was added to normalise the chloride release from the liposome vesicles at the end of the 240 second period. (b) Tip-dip experiments on human CLIC2. Single channel recordings at different membrane potentials (indicated on the left of each trace). (c) Single channel current/voltage relationship relative to four experiments similar to the examples in (b). The single channel conductance is $48.4 \pm 1.2 \mathrm{pS}$. The curve was obtained by fitting current amplitudes from -80 and $+80 \mathrm{mV}$ membrane potential by a linear regression function. At higher potentials, both negative or positive, the single channel currents rectify like other CLIC i/V curves. (d) Histogram of data from three different days in which trials were alternated at four different $\mathrm{pH}$ values. The number of successful experiments compared to the number of trials is shown above each column. The increase in the probability of observing channel openings at $\mathrm{pH} 5$ is highly significant $(\mathrm{p}<0.01)$.

\section{$\$$ undershare ${ }^{\text {Tu }}$


Table 1. Crystallographic data and refinement statistics

Data collection $^{\mathrm{a}}$

$\begin{array}{lll}\text { Crystal } & \text { Form A } & \text { Form B } \\ \text { Space group } & \mathrm{P} 2{ }_{1}{ }_{1} 2_{1} & \mathrm{P} 2{ }_{1} \\ \text { Cell dimensions }(\AA) & 44.0,74.7,79.8 & 36.0,66.9,44.1 \\ \beta\left(^{\circ}\right) & 90.0 & 99.9 \\ \text { Resolution }(\AA) & 1.85(1.92-1.85) & 1.86(1.95-1.86) \\ \text { No. of crystals } & 1 & 1 \\ \text { Temperature }(\mathrm{K}) & 100 & 100 \\ \text { Wavelength }(\AA) & 1.0875 & 1.54182 \\ \text { No. of observations } & 732050 & 102405 \\ \text { No. of unique reflections } & 23144 & 16303 \\ \text { Multiplicity } & 10.5(4.9) & 6.0(5.3) \\ \text { Data completeness }(\%) & 93.9(62.0) & 99.5(84.3) \\ I / \sigma(I) & 31.3(2.8) & 24.2(5.1) \\ R_{\text {merge }}(\%) & 6.8(30.6) & 4.7(31.5)\end{array}$


Refinement

Crystal

Non-hydrogen atoms

Protein

1836

40

Water

Resolution $(\AA)$

$R_{\text {cryst }}(\%)$

$R_{\text {free }}^{\mathrm{b}}(\%)$

Rms deviations from ideality

GSH

125

1.85

21.5

25.0

145

1.86

16.4

22.2

Bond length $(\AA)$

0.016

0.022

Bond angles $\left({ }^{\circ}\right)$

1.5

1.7

Mean B-factors $\left(\AA^{2}\right)$

Main-chain

36.8

Side-chain

37.9

17.6

Water

37.8

30.0

GSH

63.4

Residues in the most

93.0

92.1

favoured regions of the

Ramachandran plot (\%)

Residues in the disallowed

0

0.5

907

45
86
6.4
2.2


Details of the cloning, protein expression and purification have been previously described in detail. ${ }^{28}$ Briefly, CLIC2 was expressed in E. coli M15[pREP4] cells as a N-terminal Histagged fusion protein and purified by $\mathrm{Ni}$ affinity chromatography. The protein was further purified by gel filtration and then dialysed into $50 \mathrm{mM}$ HEPES pH 7.5 and $100 \mathrm{mM} \mathrm{NaCl}$ and concentrated to $7.3 \mathrm{mg} / \mathrm{ml}$ for crystal form A and $15 \mathrm{mg} / \mathrm{ml}$ in $20 \mathrm{mM}$ Tris- $\mathrm{HCl} \mathrm{pH} 7.5,50$ $\mathrm{mM} \mathrm{NaCl}$ for crystal form $\mathrm{B}$. All crystallisation experiments were carried out using the hanging-drop vapour-diffusion technique using 24-well Limbro tissue culture plates (ICN Inc.) at $19{ }^{\circ} \mathrm{C}$. Drops were formed by mixing equal volumes $(1 \mu \mathrm{l})$ of protein solution and reservoir solution. For crystal form A the reservoir solution contained 35\%-50\% (v/v) PEG 400, 100 mM Tris-HCl pH $8.0-9.2$ and 5 mM GSH. Crystals appeared after 2 to 3 days. For crystal form B the reservoir solution contained 30\% - 32\% (v/v) PEG 400, $100 \mathrm{mM}$ Tris-HCl pH 7.5 and 5 mM GSH. Crystals appeared after 3 days and were used immediately for X-ray data collection. GSH was found to be a necessary ingredient for both crystal forms.

For crystal form A, X-ray diffraction data were collected on BioCARS beamline 14ID-B at the Advanced Photon Source in Chicago, USA (Table 1). Crystals were mounted in cryo-loops (Hampton Research, CA) and transferred directly into a stream of nitrogen gas maintained at $100 \mathrm{~K}$. The structure was determined by molecular replacement using AMoRe ${ }^{48}$ with the published CLIC1 structure $^{7}$ as a probe. Subsequent rounds of model building were carried out using $\mathrm{TURBO}^{49}$ together with molecular refinement using $\mathrm{CNS}^{50}$ and REFMAC. ${ }^{51}$ The X-ray data collection and refinement statistics are shown in Table 1. Using TLS refinement $^{52,53}$ in REFMAC the model has been refined to a crystallographic $R$-factor of $21.5 \%\left(R_{\text {free }}\right.$ of $\left.25.0 \%\right)$. The final model includes residues 11 to 245 and 125 water molecules. The model is of excellent quality as judged by stereochemical parameters according to PROCHECK. $^{54}$

\section{8 undershare ${ }^{\mathrm{TM}}$


For crystal form B, X-ray diffraction data were collected in-house using a Rigaku RU200H generator equipped with mirror optics (Xenocs) and a MARResearch $345 \mathrm{~mm}$ imaging plate detector. Crystals were maintained at $100 \mathrm{~K}$ using an Oxford Cryostream. Diffraction data were integrated using MOSFLM $^{55}$ and scaled using SCALA. ${ }^{55}$ Data statistics are shown in Table 1. Molecular replacement using the previously determined CLIC2 crystal form A was carried out using PHASER. ${ }^{56}$ Subsequent rounds of model building using COOT $^{57}$ together with molecular refinement using REFMAC $5^{51}$ were carried out using all data to the highest resolution. The wild-type model has been refined to a crystallographic $R$-factor of $16.4 \%\left(R_{\text {free }}\right.$ of $\left.22.2 \%\right)$. The final model includes residues 11 to 245 and 145 water molecules (Table 1). The model is of excellent quality as judged by stereochemical parameters according to PROCHECK. ${ }^{54}$ There is one residue, Phe 83 which is located in the CLIC2 mouth region, in the disallowed region of the Ramachandran plot. The electron density for the backbone of this residue is very clear and hence it has a highly strained conformation.

CLIC2 mutants, C33A, A65C and C33A/A65C, were made by site-directed mutagenesis using the Quik Change Site-Directed Mutagenesis Kit (Stratagene) according to the manufacturer's instructions. The mutant isoforms were expressed as His-tagged-ubiquitin fusion proteins from the pHUE plasmid and purified by $\mathrm{Ni}$ affinity chromatography and ubiquitin specific protease digestion as described by Cantanzariti et al. ${ }^{58}$ Size exclusion chromatography was carried out on an Akta purifier workstation using a Superdex 75 10/300 chromatography column (GE Biosciences), with the mobile phase (Buffer A), comprising 50 $\mathrm{mM}$ sodium phosphate, $\mathrm{pH} 7.4$ and $100 \mathrm{mM}$ sodium chloride, pumped at a constant 0.5 $\mathrm{ml} /$ minute. For reducing or strongly oxidising conditions, $5 \mathrm{mM}$ DTT or $5 \mathrm{mM} \mathrm{H} \mathrm{O}_{2}$ were added to buffer A, respectively. Protein samples were diluted approximately 10 -fold to 0.5 $\mathrm{mg} / \mathrm{ml}$ in Buffer $\mathrm{A}$ and incubated at room temperature for 15 minutes prior to chromatography. Elution times were calibrated using low molecular weight protein standards $\Delta \div$ (BioRad). Gel electrophoresis was carried out using standard non-reducing SDS-PAGE. 
Chloride efflux assay was performed as described previously. ${ }^{11}$ Briefly, $400 \mathrm{~nm}$ unilamellar liposomes (soybean phosphatidylcholine:cholesterol 9:1 w:w; Sigma P-5638 and C-8662, respectively) were prepared by extrusion (Avestin Lipofast extruder) in $5 \mathrm{mM}$ sodium phosphate buffer $200 \mathrm{mM} \mathrm{KCl} \mathrm{pH} \mathrm{6.0.} \mathrm{Extravesicular} \mathrm{chloride} \mathrm{was} \mathrm{removed} \mathrm{by}$ desalting on Bio-Gel P6DG spin columns (Bio-Rad Laboratories Inc) equilibrated in $330 \mathrm{mM}$ sucrose, $5 \mathrm{mM}$ sodium phosphate at the required $\mathrm{pH}$ ( $\mathrm{pH}$ range 5 to 9). Human CLIC2 was also equilibrated into the same $\mathrm{pH}$ buffer and added to the liposomes in a total volume of 4 mL. A chloride selective electrode (Radiometer Pacific) was used to monitor the potential driven chloride efflux from the vesicles upon the addition $1 \mathrm{mM}$ valinomycin. Triton $\mathrm{X}-100$ was added to a final concentration of $1 \%$ after 240 seconds to normalize chloride release from vesicles. All experiments were carried out in the absence of reducing agent so as not to interfere with the probe.

Single-channel recordings from lipid bilayers were obtained using the tip-dip method, as previously described. ${ }^{40}$ In brief, patch clamp pipettes (Garner Glass 7052) were made using a P97 Sutter Instruments puller (Novato, CA), coated with Sylgard (Dow Corning, Midland, MI) and fire-polished to a tip diameter of 1-1.5 $\mathrm{mm}$ and 5-7 megaohm resistance. The same solution was used both in the bath and in the pipette $\left(140 \mathrm{mM} \mathrm{KCl}, 1.5 \mathrm{MgCl}_{2}, 10 \mathrm{mM}\right.$ HEPES, $\mathrm{pH}$ 6). In some experiments, the $\mathrm{pH}$ of the bath solution (trans) was altered by the addition of $\mathrm{HCl}$ or $\mathrm{KOH}$. In all experiments, as soon as the pipette tip reached the bath solution, a phospholipid monolayer (1,2-Diphytanoyl-sn-Glycero-3-Phosphocholine \#850356, Avanti Polar Lipids, Inc., Birmingham, AL) was spread on the surface. The electrode was repeatedly passed through the surface of the solution until the pipette resistance rose above 5 gigaohms. Purified recombinant human CLIC2 protein $(2 \mu \mathrm{g} / \mathrm{ml})$ was then added to the bath. An Axopatch 1D amplifier and pClamp 7 (both from Axon Instruments, Novato, CA) were used to record and analyse single-channel currents. Current recordings were digitized at 5 IOIOETSIOE 
$\mathrm{kHz}$ and filtered at $800 \mathrm{~Hz}$. Experiments carried out under reducing conditions are as described above with the exception that $5 \mathrm{mM}$ DTT was added to the bath and pipette solutions prior to the addition of protein.

${ }^{\mathrm{a}}$ The values in parentheses are for the highest resolution bin.

${ }^{\mathbf{b}} R_{\text {merge }}=\Sigma_{h k l} \Sigma_{i}\left|\mathrm{I}_{i}-<\mathrm{I}>\right| /|<\mathrm{I}>|$, where $\mathrm{I}_{i}$ is the intensity for the $i$ th measurement of an equivalent reflection with indices $h, k, l$.

${ }^{\mathbf{c}} R_{\text {cryst }}=\Sigma|| \mathrm{F}_{\text {obs }}|-| \mathrm{F}_{\text {calc }}|/ \Sigma| \mathrm{F}_{\text {obs }} \mid$, where $\mathrm{F}_{\text {obs }}$ and $\mathrm{F}_{\text {calc }}$ are the observed and calculated structure factor amplitudes respectively.

${ }^{\mathrm{d}} R_{\text {free }}$ was calculated with $5 \%$ of the diffraction data that were selected randomly and not used throughout refinement. 
Fig. 1

(a) crystal form A

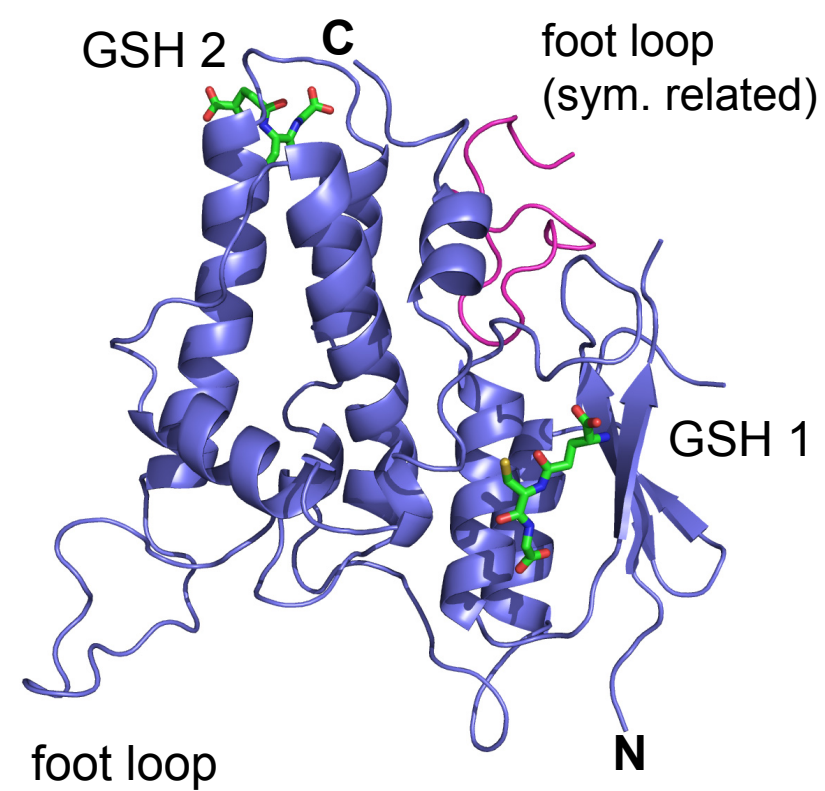

(b) crystal form B

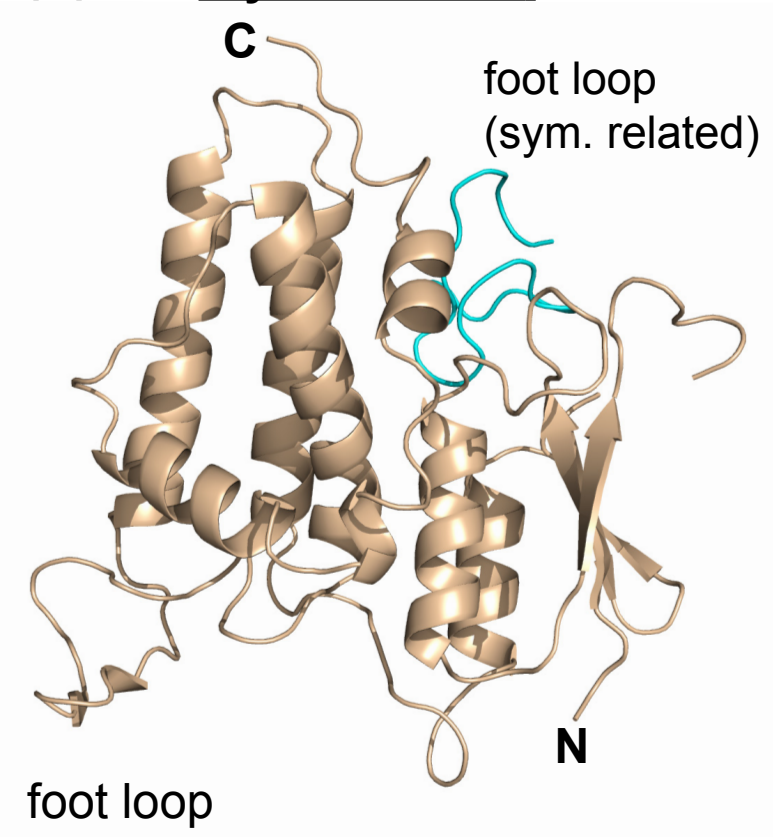


Fig. 1

(c)

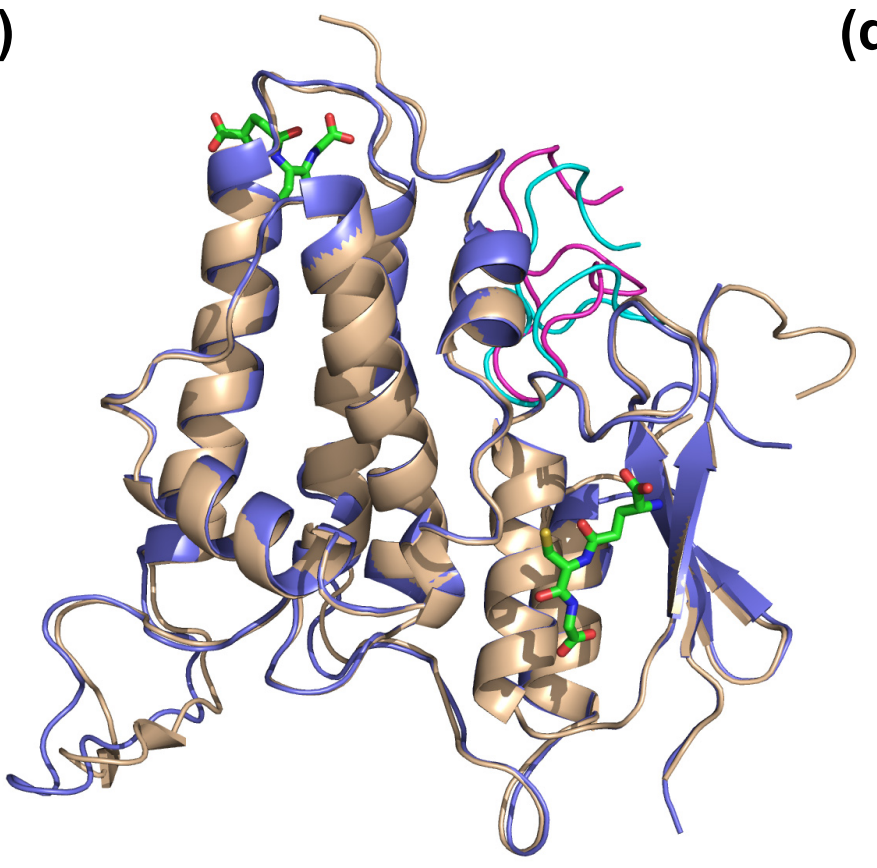

(d)

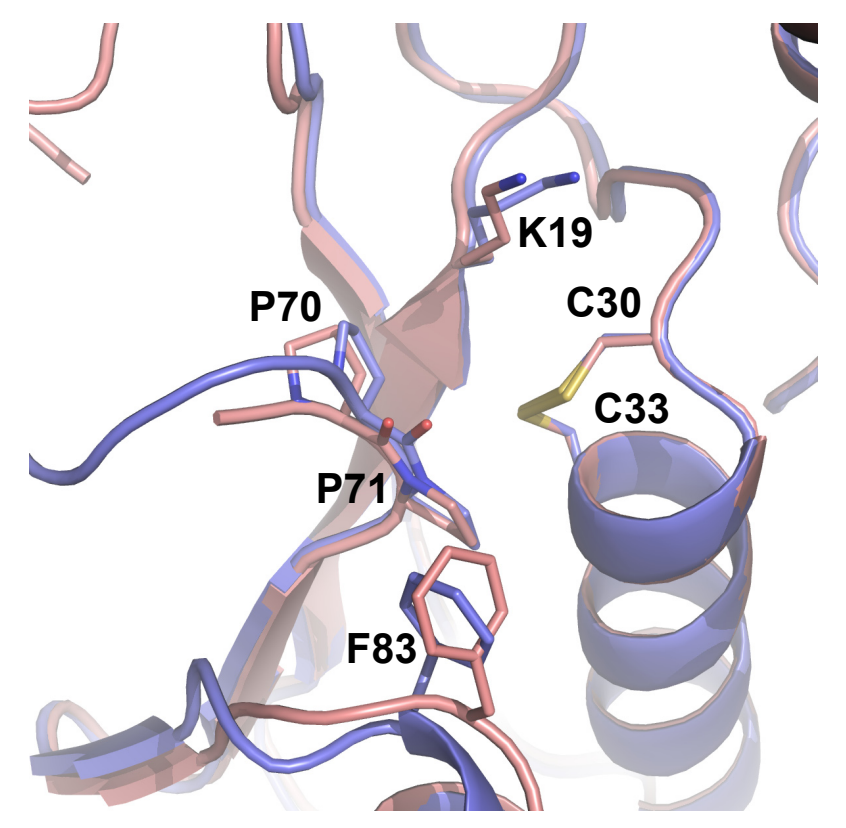


Fig. 1

(e)

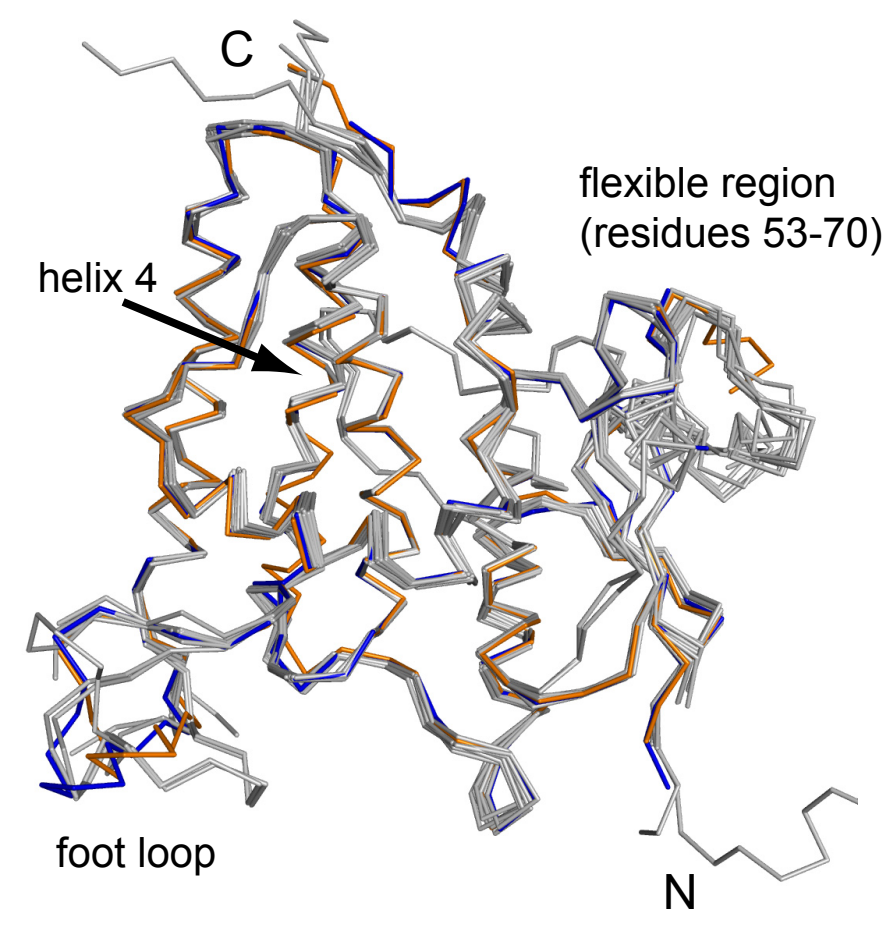

(f)

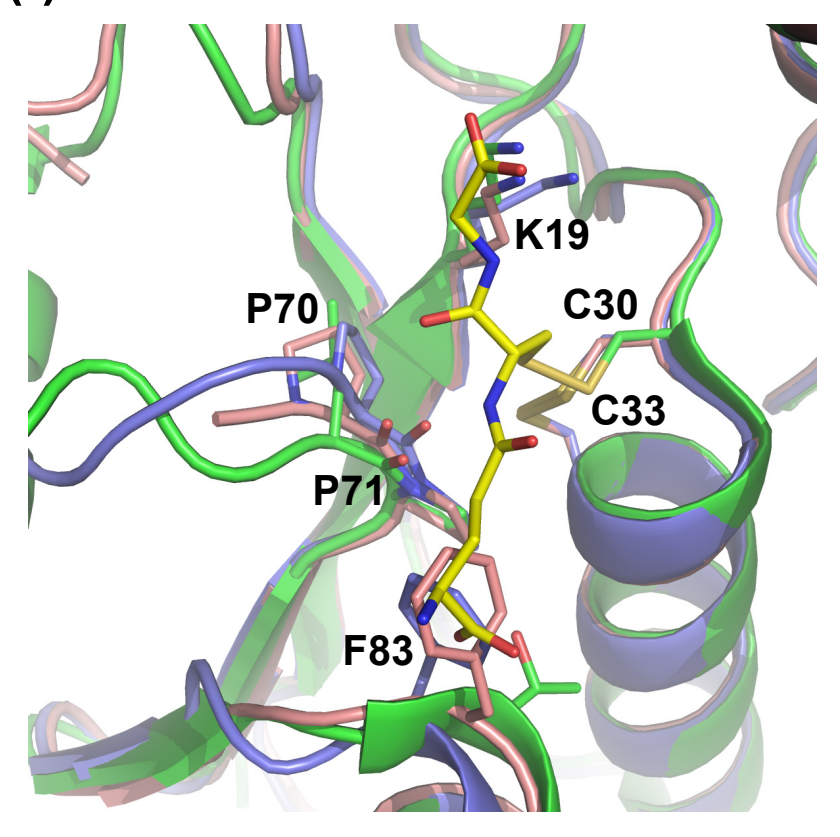

\section{4ondershare}


Fig. 2

(a)
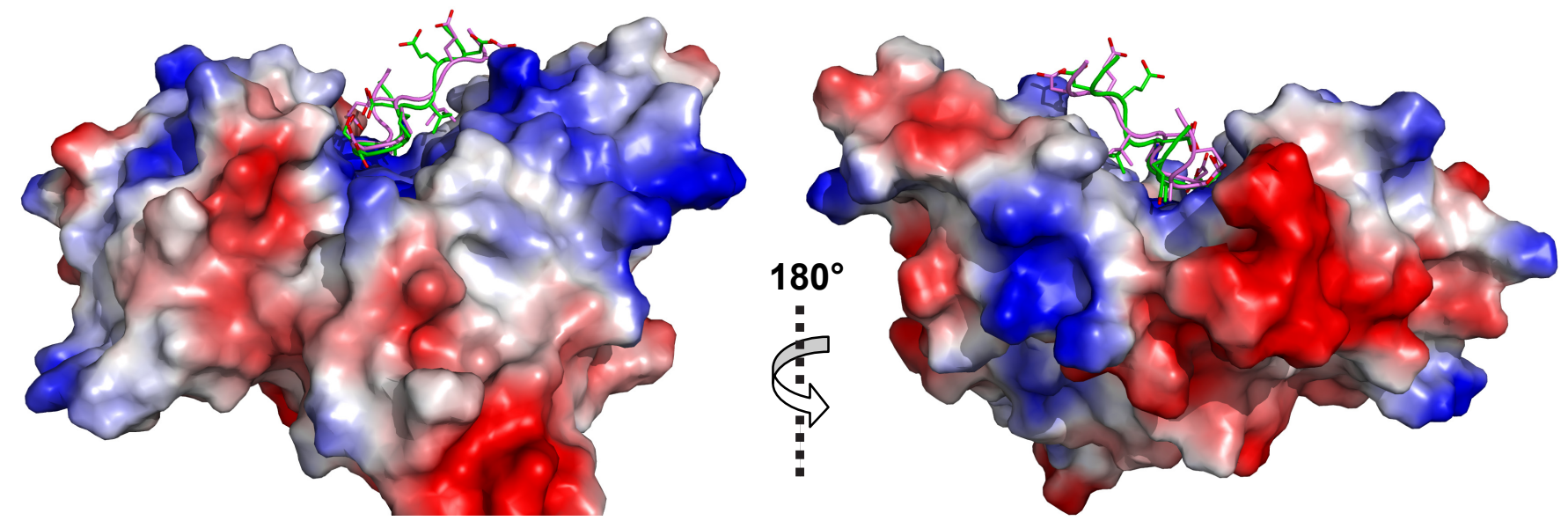

(b)

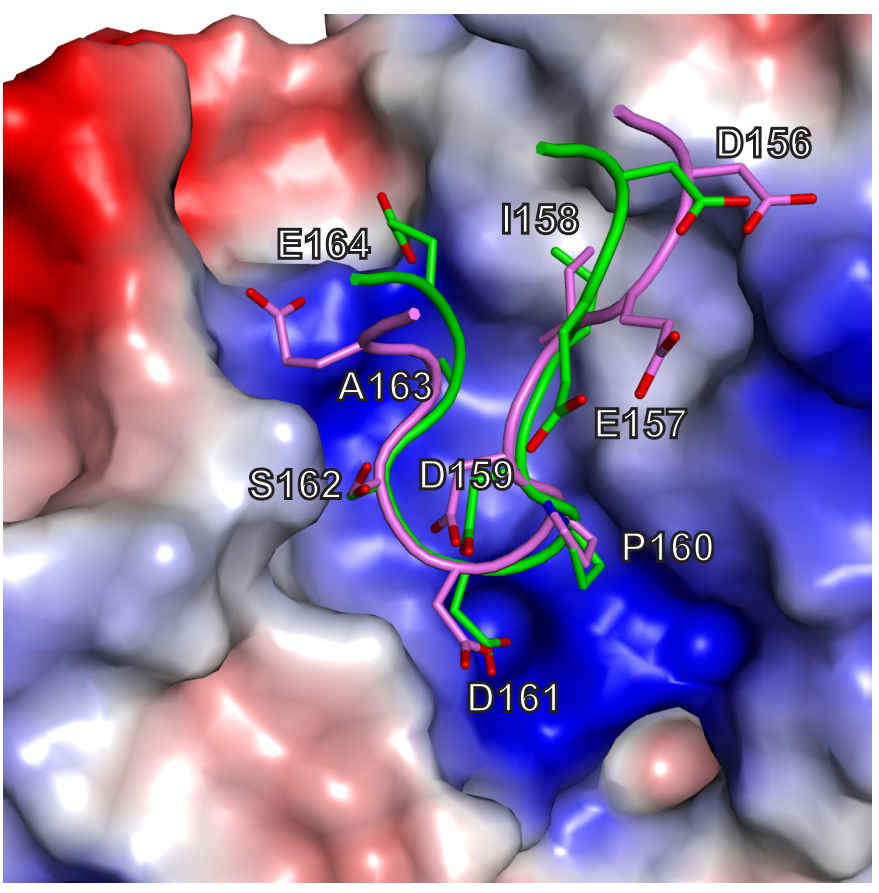

$\$ 2$ undershare ${ }^{\text {th }}$ 
Fig. 3

(a)

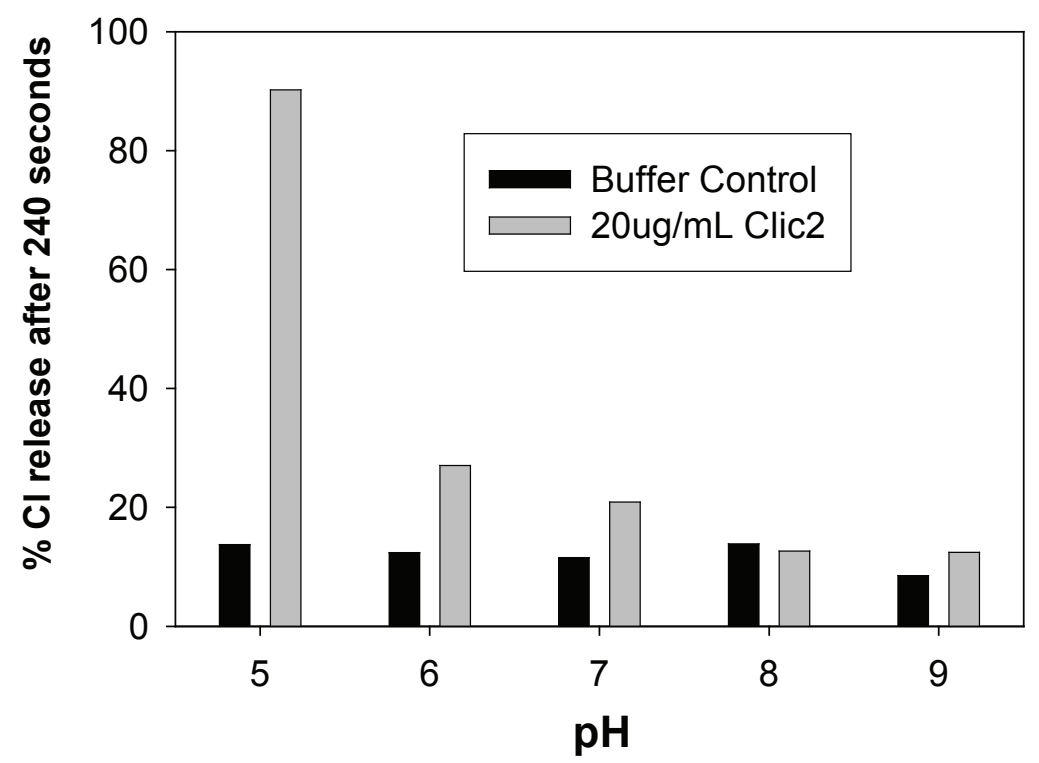

4ondershare ${ }^{\text {tw }}$ 
Fig. 3

(b)

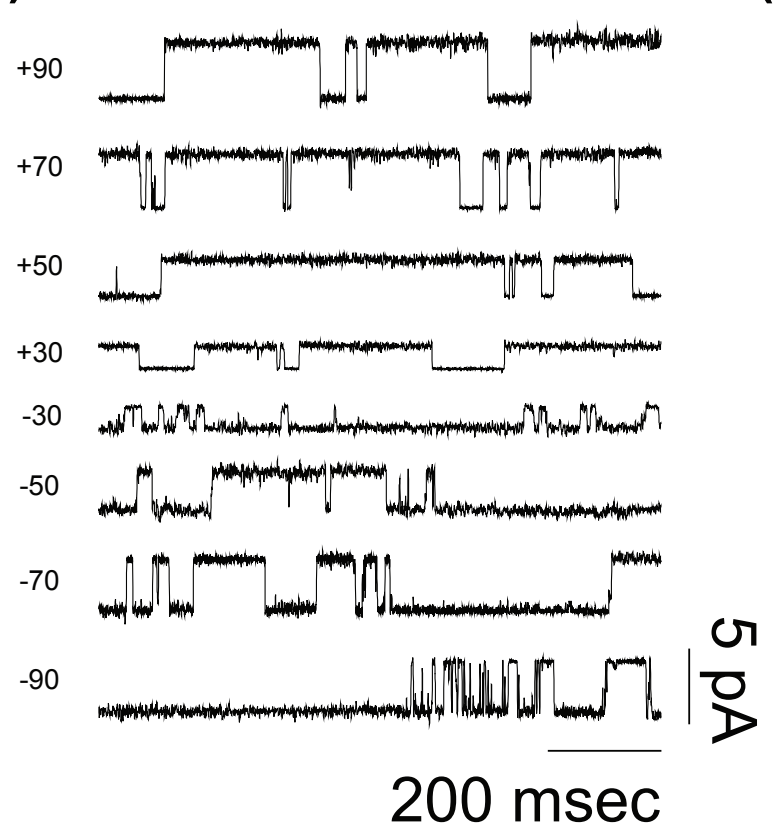

(d)

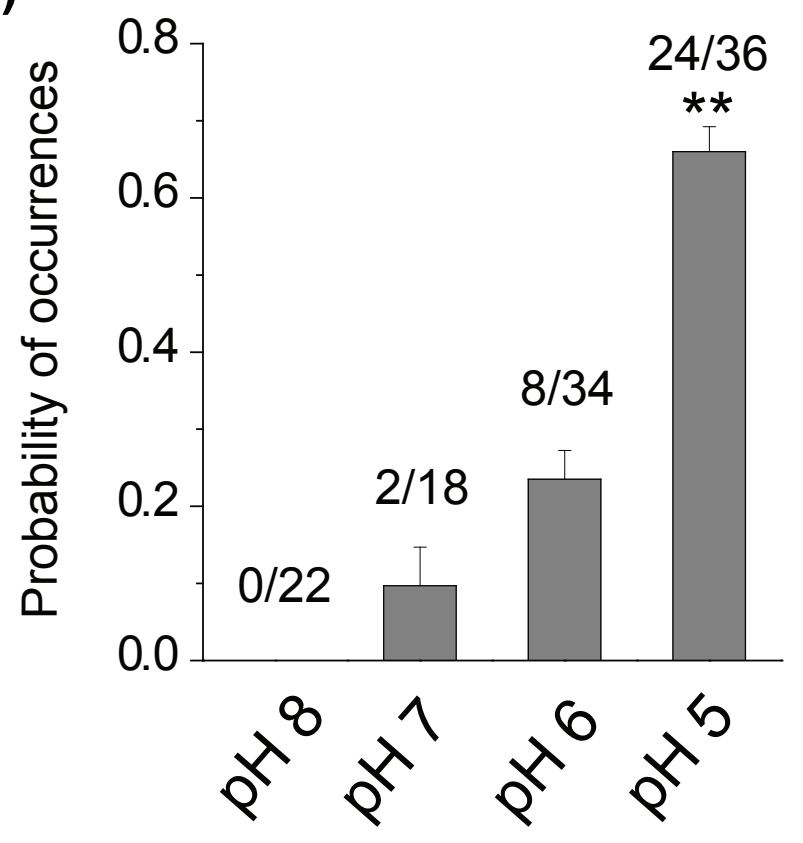

(c)

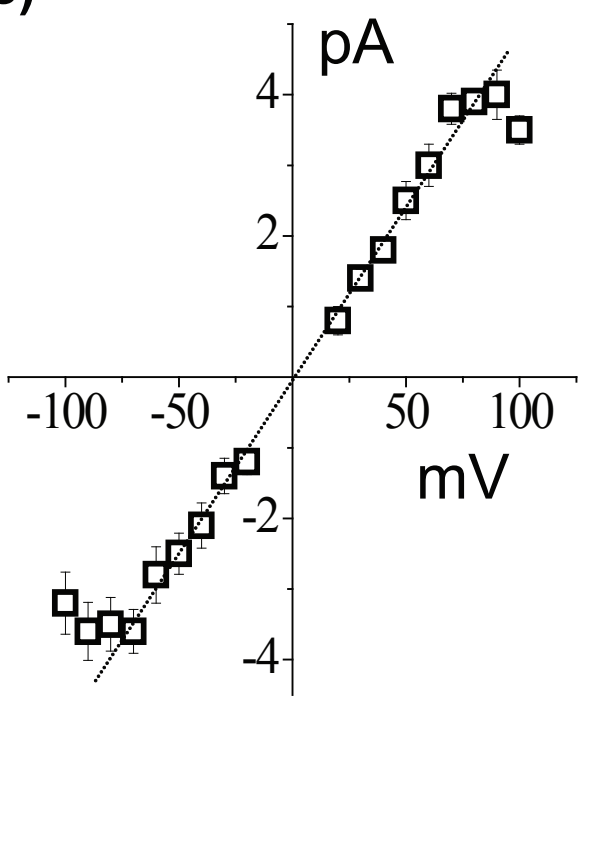

